\title{
Repetitive DNA sequence families in Hemitaxonus minomensis and $H$. athyrii (Hymenoptera; Tenthredinidae)*
}

\author{
Shoji SonOdA $^{1, * *}$, Tetsuji YAMADA ${ }^{2}$, Tikahiko NAIT0 ${ }^{3}$ \\ and Fusao Nakasuji ${ }^{1}$ \\ ${ }^{1}$ Laboratory of Applied Entomology, and \\ ${ }^{2}$ Laboratory of Genetic Engineering, Faculty of Agriculture, \\ Okayama University, Tsushima, Okayama 700 \\ ${ }^{3}$ Entomological Laboratory, Faculty of Agriculture, Kobe \\ University, Rokko, Kobe 65\%, Japan
}

(Received 18 August 1994)

\begin{abstract}
Families of the repetitive DNA sequences from Hemitaxonus minomensis and $H$. athyrii were characterized. pHMS family and pHME family in $H$. minomensis consist of tandemly arranged arrays whose basic repeat units are $260 \mathrm{bp}$ and $330 \mathrm{bp}$, respectively. pHAE family in $H$. athyrii consists of a tandemly arranged array whose basic repeat unit is $330 \mathrm{bp}$. pHMS family and pHME family occupy approximately $4.8 \%$ and $0.07 \%$ of the genome of $H$. minomensis, respectively. By contrast, in $H$. athyrii, pHAE family comprise $0.04 \%$ of the genome. Nucleotide sequence comparison of these three repetitive families showed very little homology. Southern blot hybridization using six species of Hemitaxonus showed that these repetitive families are species specific.
\end{abstract}

\section{INTRODUCTION}

Tandemly arranged repetitive DNA sequences constitute a substantial portion of the eukaryotic genome. These sequences, sometimes referred to as satellite DNA, have been found in varieties of plant and animal species in association with constitutive heterochromatin (Brutlag, 1980; Singer, 1982). Remarkable variation is observed between highly repetitive DNA sequences from different species, indicating a fast rate of evolution and dynamic component of the eukaryotic genome. Sequence comparisons between different repetitive DNA sequences from the same species indicate that sequence divergence have occurred through a random mutation or some unknown amplification mechanism (Brutlag, 1980; Singer, 1982). Much of the evolutionary history of eukaryotic genomes reflects

\footnotetext{
* The nucleotide sequence data reported in this paper will appear in the DDBJ, EMBL, and GenBank Nucleotide Sequence Databases under accession numbers of pHMS2, pHME7 and pHAE2 are D38084, D38085 and D38086, respectively.

** Corresponding author.
} 
the turnover of repetitive sequences, and speciation may be a consequence of such turnover (Rose and Doolittle, 1983).

In the previous study, we reported a family of tandemly repetitive sequences from a sawfly, Hemitaxonus japonicus (Sonoda et al., 1992). The repetitive sequences consist of the exact multiples of a basic repeat unit, having a length of $270 \mathrm{bp}$ with AT rich sequences. Only a sibling species, $H$. sasayamensis, has the homologous repetitive sequence.

Twelve species of Hemitaxonus exist in the Old World and they can be split into four species groups which respectively possess some unique features in morphological, ecological and cytological aspects (Naito, 1971). Each species group possesses sibling species, and therefore Hemitaxonus is an important material for insect genetic study. However, little work has been done on its genome structure and function at the molecular level. With the exception of Sonoda et al. (1992), there is no reports on any repetitive sequences from the genome of Hemitaxonus.

In this study, we report the cloning and characterization of the families of three repetitive DNA sequences from $H$. minomensis and $H$. athyrii.

\section{MATERIALS AND METHODS}

Insect materials

Repetitive DNA sequences analyzed in this study were cloned from $H$. minomensis and $H$. athyrii. In addition to the two species, $H$. japonicus, $H$. sasayamensis, $H$. struthiopteridis and $H$. melanogyne were used for Southern blot hybridization analysis. The taxonomical relationship deduced by Naito (1971) was shown in Table 1.

Table 1. List of Hemitaxonus species used in this study

\begin{tabular}{ll}
\hline \hline Species group & Species \\
\hline paucipunctatus & Hemitaxonus melanogyne \\
struthiopteridis & H.japonicus \\
& H. sasayamensis \\
& H. struthiopteridis \\
minomensis & H. minomensis \\
& H. athyrii \\
\hline
\end{tabular}

a. Sibling species.

Genomic DNA isolation and cloning of the repetitive DNA sequences from $\mathrm{H}$. minomensis and $\mathrm{H}$. athyrii

High molecular weight DNA was prepared from the final instar larvae of these sawflies, according to Sambrook et al. (1989).

After digestion with restriction endonucleases, $E c o$ RI or SacI for $H$. 
minomensis and $E c o \mathrm{RI}$ for $H$. athyrii, $10 \mu \mathrm{g}$ of the digests were fractionated on a preparative $1.0 \%$ agarose gel. Following ethidium bromide staining, visible bands corresponding to fragments of about 260 and $330 \mathrm{bp}$ in $\mathrm{H}$. minomensis and $330 \mathrm{bp}$ in $\mathrm{H}$. athyrii were cut out and the DNA were eluted using Prep-A-Gene Kit (Bio Rad) according to the manufacturer's recommendation. The resulting DNA was ligated into the EcoRI or SacI site of the plasmid vector pBluescript SK - (Stratagene), using Escherichia coli strain XL1-blue as a host. Recombinant clones were selected on ampicillin $(50 \mu \mathrm{g} / \mathrm{ml})$ plates containing X-gal and IPTG.

Plasmid DNA was extracted by SDS-alkaline lysis method (Sambrook et al., 1989). The inserts of the three recombinant plasmids, pHMS2, pHME7, and pHAE2, were used as probes in the subsequent experiments (see RESULTS AND DISCUSSION).

\section{Southern and slot blot hybridizations}

All restriction enzymes were used according to the manufacturer's recommendations. Completely digested DNA were fractionated on $1.0 \%$ agarose gels in TBE buffer (0.09 $\mathrm{M}$ tris base, 0.09 M boric acid, 0.002 M EDTA, pH 8.0). After ethidium bromide staining, DNA fragments were transferred onto Hybond$\mathrm{N}+$ filters (Amersham), followed by Southern blot hybridization (Southern, 1975). Filters were prehybridized at $42^{\circ} \mathrm{C}$ for $2 \mathrm{~h}$ or longer in $6 \times \mathrm{SSC}(0.9 \mathrm{M} \mathrm{NaCl}, 0.09$ $\mathrm{M}$ sodium citrate), $50 \%$ formamide, $0.5 \%$ SDS, $5 \times$ Denhardt's solution $(0.1 \%$ BSA fraction $\mathrm{V}, 0.1 \%$ polyvinylpyrolidone, $0.1 \%$ Ficoll 400 ), and $0.1 \mathrm{mg} / \mathrm{ml}$ of sonicated herring DNA. Hybridization was carried out by the addition of ${ }^{32} \mathrm{P}$-labeled probes and incubated for $12 \mathrm{~h}$. DNA was labeled using Random Primed Labeling Kit (Boehringer). After hybridization, the membrane was washed twice with $2 \times$ SSC, $0.1 \%$ SDS at room temperature followed by washing with $1 \times$ SSC, $0.1 \%$ SDS at $60^{\circ} \mathrm{C}$. After drying, the membrane was exposed to X-ray film (KODAK O-MAT) for autoradiography.

Slot blots were obtained by spotting variable amounts of denatured genomic DNA onto Hybond-N + filters; denatured pHMS2, pHME7, and pHAE2 were used as positive controls. Prehybridization and hybridization conditions and washes were those used for genomic Southern blot hybridization and the specific radioactivity was determined using FUJIX BAS 2000 System.

\section{DNA sequencing}

The nucleotide sequences were determined by chain termination method (Sanger et al., 1977) using Sequenase Version 2.0 (United States Biochemical). Sequence data were analyzed using GENETYX (Software Development). 


\section{RESULTS AND DISCUSSION}

Molecular structure of repetitive sequences

When genomic DNA from $H$. minomensis was digested with Sac I, fractionated on $1.0 \%$ agarose gel and stained with ethidium bromide, prominent bands of 260 bp and the multimers were observed. EcoRI digests of $H$. minomensis and $H$. athyrii also showed bands of $330 \mathrm{bp}$. The three bands were cut out and their DNA cloned in the plasmid vector pBluescript SK-. From each experiment we selected clones for subsequent analysis: pHMS2 and pHMS3 contained $260 \mathrm{bp}$ Sac I fragments from H. minomensis, pHME7 contained a $330 \mathrm{bp} E c o$ RI fragment from $H$. minomensis, pHAE2, pHAE3 and pHAE7 contained $330 \mathrm{bp}$ EcoRI fragments from $H$. athyrii.

To determine whether the cloned repetitive DNA sequences were dispersed or tandemly repetitive sequences, genomic DNA of $H$. minomensis and $H$. athyrii was examined by Southern blot hybridization (Fig. 1). pHMS2 insert hybridized to multimers of a basic $260 \mathrm{bp}$ unit that were generated by digestion with Sac I, Dra I, and AluI, indicating that the cloned DNA belonged to a tandemly repetitive DNA sequences. The strongest intensity of the hybridization bands was at dimer $(520 \mathrm{bp})$ position and the band intensity gradually declined with an increase of the length. Signals on the Sau3AI pattern did not correspond to the basic 260 bp unit because, as shown below by sequencing, most units have more than one Sau3AI site. Hybridization patterns of the EcoRI and Ssp I digests revealed that a fraction of the homologous DNA remained undigested, since DNA bands corresponding to high molecular weight multimers which showed nearly the same mobility as undigested DNA were observed (Fig. 1). This signifies the existence of certain subfamilies that have escaped from homogenization which occur through mechanisms such as gene conversion and unequal crossing-over.

Hybridization pattern with ${ }^{32} \mathrm{P}$-labeled pHME7 insert is shown in Fig. 2. EcoRI internally cleaved all the repeat units, resulting in a monomeric unit of approximately $330 \mathrm{bp}$ and the respective dimer and trimer. The band intensity gradually declined with an increase of the length. This hybridization pattern is indicative of a tandemly arranged repetitive DNA family. Sac I digests revealed the hybridization pattern showing that the majority of homologous DNA remained undigested. Signals on the Ssp I pattern did not correspond to the basic $330 \mathrm{bp}$ unit because, as shown below by sequencing, most units have more than one $S s p$ I site. Dra I, Sau3AI, and Alu I digests displayed deviations of the regular ladder pattern. These hybridization patterns appear to represent the repeat units within the array that contained some RFLP (restriction fragment length polymorphism).

pHAE2 insert hybridized to multiple genomic EcoRI and Sau3AI fragments from $H$. athyrii DNA that were multimers of a basic $330 \mathrm{bp}$ unit, indicating that the cloned DNA also belonged to a tandemly repetitive DNA sequences (Fig. 3). 


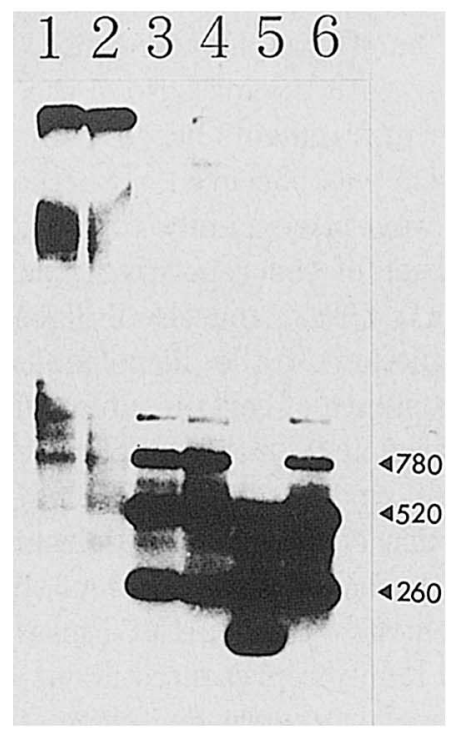

Fig. 1. Southern blot analysis of $H$. minomensis genomic DNA digests with different restriction enzymes using the insert of pHMS2 as a probe. Genomic DNA extracted from $H$. minomensis were digested with restriction enzymes (lane 1, Eco RI; 2, Ssp I; 3, Sac I; 4, DraI; 5, Sau3AI; 6, AluI), sizefractionated on $1.0 \%$ agarose gel, blotted on Nylon membrane and hybridized with the probe. The numbers at the far right indicate the size of DNA fragments in bp.

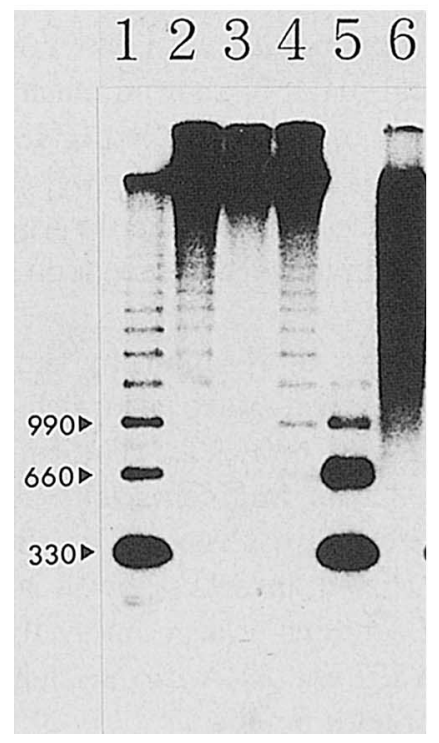

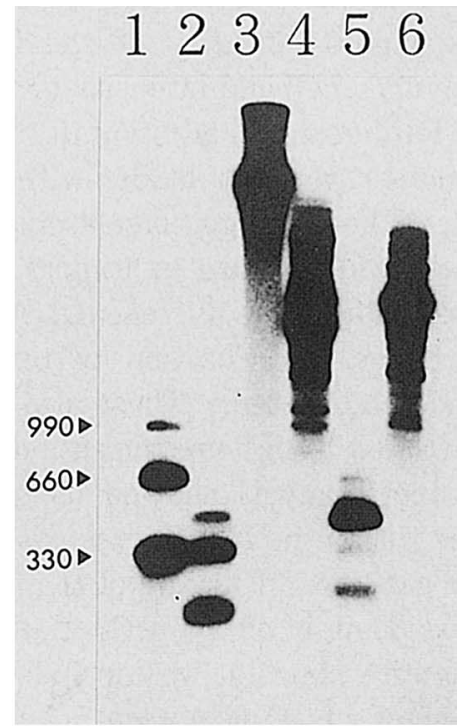

Fig. 2. Southern blot analysis of $H$. minomensis genomic DNA digests with different restriction enzymes using the insert of pHME7 as a probe. Genomic DNA extracted from $H$. minomensis were digested with restriction enzymes (lane 1, Eco RI; 2, Ssp I; 3, Sac I; 4, DraI; 5, Sau3AI; 6, AluI), sizefractionated on $1.0 \%$ agarose gel, blotted on Nylon membrane and hybridized with the probe. The numbers at the far left indicate the size of DNA fragments in bp.

Fig. 3. Southern blot analysis of $H$. athyrii genomic DNA digests with different restriction enzymes using the insert of pHAE2 as a probe. Genomic DNA extracted from $H$. athyrii were digested with restriction enzymes (lane 1, EcoRI; 2, Ssp I; 3, Sac I; 4, Dra I; 5, Sau3AI; $6, A l u \mathrm{I})$, size-fractionated on $1.0 \%$ agarose gel, blotted on Nylon membrane and hybridized with the probe. The numbers at the far left indicate the size of DNA fragments in bp. 
The strongest intensity of the hybridization band was at monomer (330 bp) position and the band intensity gradually declined with the increase of the length. In $E c o$ RI digests, in addition to the main ladder of fragments based upon the 330 bp monomer, a minor ladder with a different size was observed. Furthermore, $E c o$ RI, although digesting largely within the repetitive family to bring about monomer and low-size multimers, shows a subset of the repetitive family that remains undigested as relic DNA. Ssp I, SacI, Dra I, and Alu I digests also revealed the hybridization patterns that a fraction of the homologous DNA remained undigested. These also signify the existence of certain subfamilies that have escaped from homogenization as are the cases in $H$. minomensis.

Slot blot analysis allowed us to estimate the amounts of pHMS and pHME families within the $H$. minomensis genome and that of pHAE family within the $H$. athyrii genome. Fifty ng of $H$. minomensis genomic DNA gave about the same signal as $30 \mathrm{ng}$ of entire pHMS2 plasmid as a control. Since pHMS2 insert is $8 \%$ of the entire plasmid, we concluded that pHMS family represented about $4.8 \%$ of the genome of $H$. minomensis. Using the same procedure and calculations, it was estimated that pHME family comprises about $0.07 \%$ of the genome of $H$. minomensis and pHAE family comprises about $0.04 \%$ of the genome of $H$. athyrii.

\section{Sequence analysis of the clones}

pHMS family, represented by the clones pHMS2 and pHMS3, was composed of a repetitive DNA sequences with a length of $255 \mathrm{bp}$ and $254 \mathrm{bp}$, respectively (Fig. $4 \mathrm{~A})$. These repetitive sequences showed a homology of $93 \%$ with average AT content of $69 \%$. Sequence comparison of the two repetitive sequences implicated that variations might have been occurred due to point mutations and deletions. The repetitive sequence in pHMS2 could be further divided into two smaller sub-domains between nucleotides 1 and 128 and 129 and 255 with $78 \%$ homology (Fig. 5A). Repeat units of the clones, pHMS2 and pHMS3, showed much higher homology (93\%) than between two adjacent internal repeats (78\% in pHMS2, 76\% in pHMS3). We consider, with respect to the evolution of pHMS family, that the repeat unit might probably have been arisen by duplication and the divergence of an ancestor unit with approximately $130 \mathrm{bp}$. An inverted subrepeat in pHMS family is also shown in Fig. $4 \mathrm{~A}$.

The pHME (Fig. 4B) and pHAE (Fig. 4C) families, represented by the clones pHME7 and pHAE2, pHAE3 and pHAE7, respectively, are also rich in AT sequences. pHME7 insert is $334 \mathrm{bp}$ long that contains $68 \%$ AT. The repetitive sequences in pHAE2, pHAE3, and pHAE7 are $329 \mathrm{bp}$ fragments with AT rich (65\%) sequences. pHME7 insert contains imperfect direct repeats as shown in Fig. 4B. pHAE family represented by three cloned inserts showed average homology of $97 \%$. The 329 bp insert of pHAE2 contains a large internal subrepeat of $98 \mathrm{bp}$ between nucleotides 17 and 114 and 197 and 294 with 76\% homology (Fig. 5B). No inverted repeats were observed in both families. 


\section{A}

PHMS2 CATTTTGCGG GAAAATCACC TATTTTTGAC CGAATTTTGG ACAATTTTTT TTTTTGCCTA 60

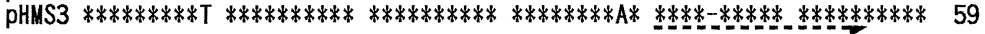

pHMS2 AAAACGACCA TATCTTGGCT TCTATCAATC GTACGTTATT TTTATCCATA TTTTCGGTTT 120 pHMS3 $* * * * * * * * * * * * * * * * * A * * * * * * * * * * * * * * * * * * * * * *$ * ***************** 119

pHMS2 TGTGAGATCA TTTTGCATAA GAATCACCCA TTTTTGACTG AATTTTTGAC ATTTTTTTTT 180

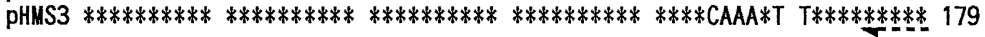

PHMS2 TTTTCTAAAA ATGATCATAT CTTCGCTCCT GTAGATTCGA CGTCATTTTC AACTATATTT 240 pHMS3 $* * * C * * * G * * * C *$ CT**** ******************************T $* * * * * * * * * * 239$

PHMS2 TCGGTACTTT GAGCT

pHMS3 ****************

B

AATTCTGTAT TITCTCAGAA TAGACTCTGA AAAATGGAAT CGCAATACCG CTATTTTATG 60

CAAATCAATC TTGTGGATTC GAGGACGTTA GTAAAATAAT GTGAGAGCAA TAAAAAAAAG 120

AAATCAATGA TTTCATACAT TTTCATTTCA TGCAATTTTT TTTTTCACGA AAATATTCCG 180

AGGAATGGGT TTGTGACGAC ACTTTCTCAT GAAGAATGAT TCCAGTAATT CGAAAATATG 240

ATCAAAATCA CCGTACGACC AAAAGAACTG GAGTATTCGA TGATTTCATG TCTTTTCGTA 300

TTTCACGCAA AATATTTCAA AAAATGGGTC GCTG

C

PHAE2 AATTCTATCG CCTATAGGTG TTTCTTATGC AGAATTATCT CAGGAATGCA ACAAACCGAG 60 pHAE3 $* * * * * * * * * * * * * * * * * * * * * * * * * * * * * * * * * * * * * * C * * * * * * * * * * \mathrm{G} * * * * * * * * *$

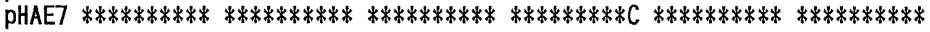

PHAE2 TCTCATTGTT GTGCGATACT CAGGGAATAA GATATCGACA ATTTTCAGGA TTCTCGATTT 120 pHAE3 $* * * * * * * * * * * * * * * * * * * *$ **************************************** pHAE7 ************************************************************

PHAE2 TCGCAAAAAT CATAAGGGT TAGCCTTACT TTTTTTTCGA CGAAAAAAA AGTTGACTGG 180 pHAE3 ****************************** C*************G**********T****

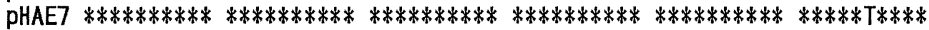

PHAE2 AATTTATTTC TTTCCGGGTG TTTCTTGTAG AGAATAATCT CACGAATCCA AAAAACGCAG 240

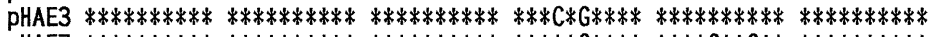
pHAE7 $* * * * * * * * * * * * * * * * * * * * * * * * * * * * * * * * 6 * * * * * * * \mathrm{G} * \mathrm{G} * * * * * * * * * * * *$

PHAE2 TCTCGTTGTT GTGCGATAAT TAGGAGAAAA GATATTCAAG ATTCACTGAA TTTTTTTATT 300

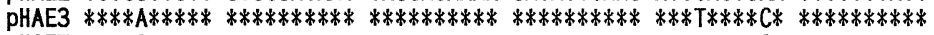
PHAE7 ****A*******************************************C***********

PHAE2 TTTCTAAAAA TCGGAGAAAT GTAGGTCAG

PHAE3 $* * * * * * * * * * * * * * * * * * * * * * * * * * * * * *$

PHAE7 ******************************

Fig. 4. Nucleotide sequences of the inserts of pHMS2 and pHMS3 (A), pHME7 (B), and pHAE2, pHAE3 and pHAE7 (C). Direct and inverted repeats are shown by solid and broken arrows, respectively. Identical nucleotides are represented by asterisks. A dash indicates a deletion. 


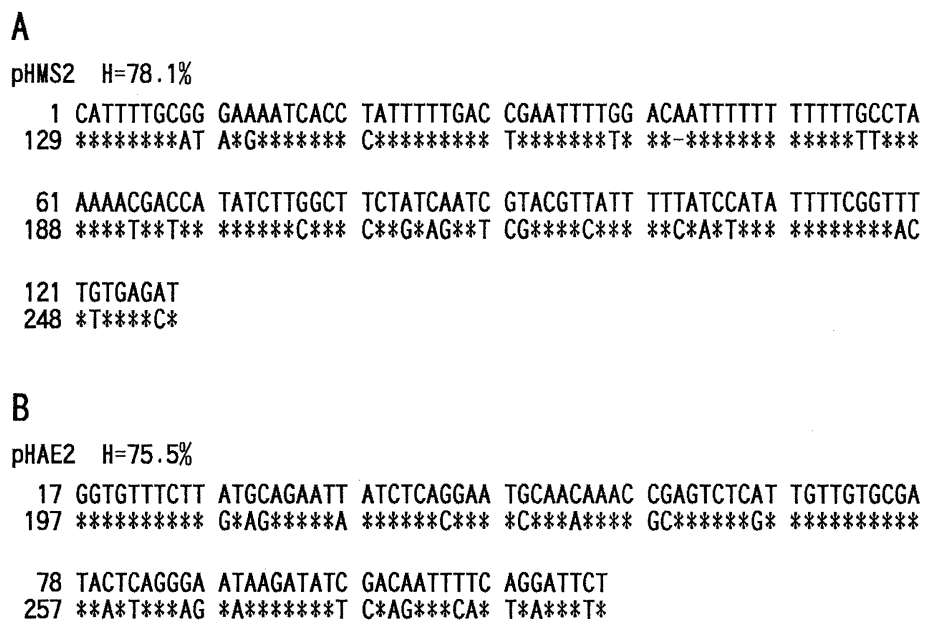

Fig. 5. Sequence comparisons between two internal subrepeats from pHMS2 (A) and pHAE2 (B) inserts. The percentage homology $(\mathrm{H})$ is given for each alignment.

The nucleotide sequences of the three families analyzed above did not show any significant homology among them, indicating that they represented different repetitive DNA families. Including a family of tandemly repetitive sequences from $H$. japonicus (Sonoda et al., 1992), no nucleotide sequences with significant homology were identified by a EMBL Data Library search. Despite the difference in base constitution, the size of the repeat units is strongly conserved between different repetitive families from different sawfly species. The conservation of the size of the repeat unit among sawfly species implicates the hypothesis that these repetitive sequences may have some common property. The size of the repeat units from the alphoid satellite and from several other plant satellites is conserved in between 150 and $180 \mathrm{bp}$ (Brutlag, 1980; Schmidt and Heslop-Harrison, 1993). In these cases, there exists a striking similarity between the size of the repeat unit and the size of the DNA in a chromatin nucleosome containing tandemly repetitive sequences, suggesting their involvement in determining chromatin structure (Grellet et al., 1986; Martinez-Zapater et al., 1986; Schmidt and Heslop-Harrison, 1993).

\section{Evolution and distribution in the genus}

To determine whether sequences homologous to pHMS and pHME families from $H$. minomensis and $\mathrm{pHAE}$ family from $H$. athyrii were also present in other related species, the genomic DNA from $H$. minomensis, $H$. athyrii, $H$. japonicus, $H$. sasayamensis, $H$. struthiopteridis, $H$. melanogyne were digested with Sac I (for pHMS family) or Eco RI (for pHME and pHAE families), size-fractionated on $1.0 \%$ agarose gel and hybridized with ${ }^{32} \mathrm{P}$-labeled probes of the three representative repetitive sequences. The results showed a lack of sequences homologous to 

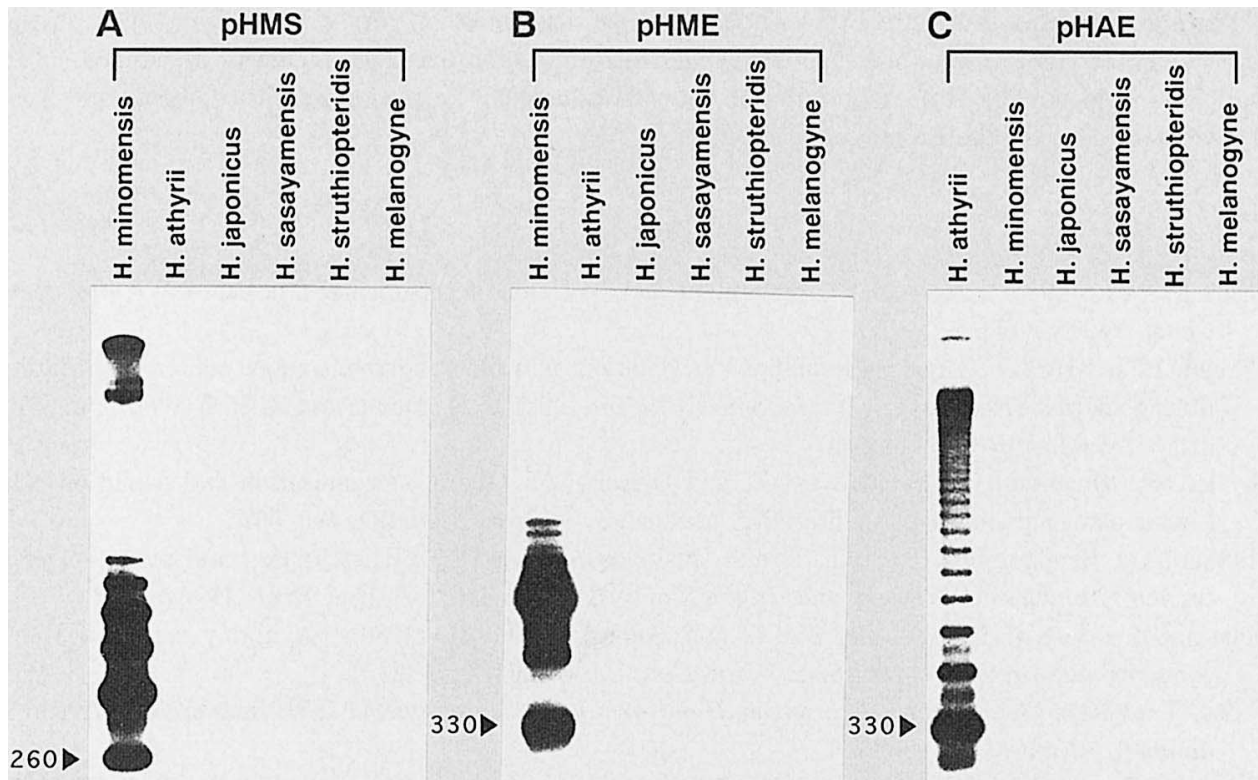

Fig. 6. Presence of the repetitive DNA sequences in the genus Hemitaxonus genomic DNA. The genomic DNA from $H$. minomensis, $H$. athyrii, $H$. japonicus, $H$. sasayamensis, $H$. struthiopteridis and $H$. melanogyne were used for Southern blot analysis. (A) SacI digests probed with pHMS2 insert. (B) Eco RI digests probed with pHME7 insert. (C) Eco RI digests probed with pHAE2 insert. The numbers at the far left indicate the size of DNA fragments in bp.

the three families in any of the related species (Fig. 6). It is considered that tandemly repetitive DNA sequences occur through mechanisms such as rolling circle replication or unequal crossing over, and diverged rapidly by accumulation of mutations or through process such as conversions and crossing over (Singer, 1982; Halldén et al., 1987). The function of repetitive DNA sequences is highly speculative. The association of tandemly repetitive sequences with centromeric and telomeric heterochromatin has led to a suggestion that they might play a role in chromosomal pairing (Flavell, 1982; Rose and Doolittle, 1983). On the other hand, Orgel and Crick (1980) suggested that repetitive sequences are parasitic element and have no meaningful function.

Analyses of $H$. minomensis and $H$. athyrii in this study and $H$. japonicus (Sonoda et al., 1992) revealed the presence of families of tandemly repetitive sequences whose repeat units are similar in size but consist of different nucleotide sequences. This fact suggests that other Hemitaxonus species also have such repetitive sequences. Further comparative molecular studies on such repetitive sequences might provide insight into the evolution of genomes and species in Hemitaxonus. 
The authors thank Associate Professor Y. Ichinose, Okayama University, and all members of the Laboratory of Genetic Engineering, Okayama University, for excellent technical assistance. The work was supported by Grants from the Ministry of Education, Science and Culture, Japan and from the Japan Society for the Promotion of Science.

\section{REFERENCES}

Brutlag, D. L. (1980). Molecular arrangement and evolution of heterochromatic DNA. Annu. Rev. Genet. 14, 121-144.

Flavell, R. B. (1982). Sequence amplification, deletion, and rearrangement-major source of variation during species divergence. In: Genome Evolution (eds.: G. A. Dover and R. B. Flavell), pp. 301323. Academic Press, London.

Grellet, F., Delcasso, D., Panabieves, F. and Delseny, M. (1986). Organization and evolution of a higher plant alphoid-like satellite DNA sequence. J. Mol. Biol. 187, 495-507.

Halldén, C., Bryngelsson, T., Säll, T. and Gustavsson, M. (1987). Distribution and evolution of a tandemly repeated DNA sequence in the family Brassicaceae. J. Mol. Evol. 25, 318-323.

Martinez-Zapater, J. M., Estelle, M. A. and Somerville, C. R. (1986). A highly repeated DNA sequence in Arabidopsis thaliana. Mol. Gen. Genet. 204, 417-423.

Naito, T. (1971). A revision of the genus Hemitaxonus in the old world, I (Hymenoptera, Tenthredinidae). Kontyu 39, 19-28.

Orgel, L. E. and Crick, F. H. C. (1980). Selfish DNA: the ultimate parasite. Nature 284, 604-607.

Rose, M. R. and Doolittle, W. F. (1983). Molecular biological mechanisms of speciation. Science 220, 157-162.

Sambrook, K., Fritsch, E. F. and Maniatis, T. (1989). Molecular Cloning: A Laboratory Manual. Cold Spring Harbor Laboratory Press, New York.

Sanger, F., Nicklen, S. and Coulson, A. R. (1977). DNA sequencing with chain-terminating inhibitors. Proc. Natl. Acad. Sci. USA 74, 5463-5467.

Schmidt, T. and Heslop-Harrison, J. S. (1993). Variability and evolution of highly repeated DNA sequences in the genus Beta. Genome 36, 1074-1079.

Singer, M. F. (1982). Highly repeated sequences in mammalian genomes. Int. Rev. Cytol. 76, 67112.

Sonoda, S., Yamada, T., Naito, T. and Nakasuji, F. (1992). A family of highly repeated DNA sequences of the fern sawfly Henitaxonus japonicus complex (Hymenoptera: Tenthredinidae). Appl. Entomol. Zool. 27, 399-405.

Southern, E. M. (1975). Detection of specific sequences among DNA fragments separated by gel electrophoresis. J. Mol. Biol. 98, 503-517. 